\title{
Dead-Space Theory Predictions of Excess-Noise Factor, Breakdown Voltage, and Frequency Response for Thin Avalanche Photodiodes
}

\author{
Majeed M. Hayat ${ }^{a, 1}$, Mohammad A. Saleh ${ }^{b}$, Ohhyun Kwon ${ }^{c}$, \\ Bahaa E. A. Saleh ${ }^{d}$, and Malvin C. Teich ${ }^{e}$ \\ $a, b, c$ The Electro-Optics Program, University of Dayton, Dayton, $\mathrm{OH}$ \\ d,e Department of Electrical and Computer Engineering, Boston University, Boston, MA
}

\begin{abstract}
The dead-space carrier multiplication theory properly predicts the reduction in the excess noise factor in a number of APDs. The theory is applied to measurements, obtained from J. C. Campbell and collaborators at the University of Texas, for InP, InAlAs, GaAs, and AlGaAs APDs with multiplicationregion widths ranging from $80 \mathrm{~nm}$ to $1600 \mathrm{~nm}$. A refined model for the ionization coefficients is reported that is independent of the width of the device multiplication region of each device. In addition, in comparison to predictions from the conventional multiplication theory, the dead-space multiplication theory predicts a reduction in the mean bandwidth as well as a reduction in the power spectral density of the impulse response. In particular, it is shown that the avalanching noise at high-frequencies is reduced as a result of the reduction of the multiplication region width.
\end{abstract}

Keywords: Avalanche photodiode, dead space, impact ionization, excess noise factor, avalanche breakdown down, frequency response.

\section{Introduction}

It is well known that for avalanche photodiodes (APDs) with thin multiplication regions, the conventional (McIntyre) carrier-multiplication theory ${ }^{1}$ does not correctly predict the reduction in the noise characteristics. This reduced noise characteristic of thin APDs has been established by both experiments and Monte-Carlo simulations. ${ }^{2-6}$ The inadequacy of the conventional theory to explain the noise reduction has been attributed primarily to the inability of the conventional multiplication model to capture the dead-space effect. The dead space is the minimum distance a newly-created carrier must travel before it is capable of effecting a new impact ionization.

Using excess-noise versus mean-gain data for thin GaAs, AlGaAs, InP, and InAlAs APDs, collected by J. C. Campbell and collaborators at the University of Texas at Austin, it has been shown that the dead-space multiplication theory (DSMT), developed earlier by Hayat et al., ${ }^{7}$ resolves the discrepancy

\footnotetext{
${ }^{1}$ E-Mail: mhayat@udayton.edu, Telephone (937) 229-4521, Fax: (937) 229-2097
} 
between experimental data and the conventional-theory predictions. ${ }^{9}$ Other researchers ${ }^{10,11}$ have also reached similar conclusions using a modified version of the original dead-space theory. ${ }^{7,8}$ In this paper, the DSMT is used in conjunction with measurements of the gain and excess noise factor to establish a refined width-independent model for the ionization coefficient of each carrier capable of effecting an impact ionization. This refinement is accomplished by means of optimizing over the carrier threshold energies. The model predicts the ionization coefficients as a function of the electric field, within the confines of a single set of parameters which is suitable for all multiplication-region widths.

In this paper, we use the above refined ionization coefficients in the DSMT model to provide excessnoise-factor versus mean-gain curves that are in excellent agreement with those measured for each device, regardless of the multiplication-region width. In addition, an extension of the DSMT is developed that allows the computation of the autocorrelation function of the APD impulse response. This extension involves developing six recurrence equations, which are derived according to the same renewal-theory principles used in deriving the mean and the variance of the impulse response. ${ }^{12}$ The extracted widthindependent ionization coefficients are then used in conjunction with the theory to predict the statistics of the frequency response of thin APDs. Our results indicate that the application of the conventional McIntyre theory tends to overestimates the 3-dB bandwidth of thin APDs. Finally, the theory can also be used to predict the breakdown voltage for thin devices. Preliminary results indicate that the application of the conventional McIntyre theory tends to underestimate the breakdown voltage in thin APDs.

\section{Dead-Space Multiplication Theory}

In this section, we review the relevant aspects of the dead-space multiplication theory that will allow the prediction of the excess noise factor, the statistics of the bandwidth, and the breakdown voltage. Consider an electron-injected APD, and suppose that the multiplication region is of width $w$. The nonlocalized impact ionization theory ${ }^{13}$ assumes that each newly created carrier must travel a fixed distance, called the dead space, within the high-field multiplication region before it is capable of impact ionizing. The dead space for electrons and holes can be calculated apriori through knowledge of the electric field and the ionization thresholds. Moreover, threshold energies for various materials are available in the literature. After traversing the dead space, a carrier may impact ionize with a probability rate which is characterized by the ionization coefficients. Since these ionization coefficients are associated with carriers that have traversed the dead space, they are the physical ionization coefficients. The physical ionization coefficients are different from the effective ionization coefficients computed from conventional localized models which do not account for dead space.

The dead-space multiplication theory (DSMT) is an analytical model that facilitates the computation of the gain, the excess noise factor, the impulse response statistics, and the breakdown voltage. Furthermore, the DSMT can be used to extract the physical ionization coefficients from measurement. This can be done by fitting the gain-versus-noise measurement to that predicted by the DSMT and adjusting the ionization coefficients until a good fit is obtained. It has been shown ${ }^{9}$ that these physical ionization coefficients are material specific and do not depend on the the thickness of the multiplication region. The effect of reducing the multiplication region on the device noise characteristics is accommo-

dated by the theory when these physical coefficients are used in conjunction with DSMT to determine the APD noise and response-time characteristics. 


\subsection{Gain noise}

The gain and the excess noise factor can be calculated by solving the following set of recurrence equations ${ }^{7}$ :

$$
\begin{aligned}
& z(x)=\left[1-\int_{0}^{w-x} h_{e}(\xi) d \xi\right]+\int_{x}^{w}[2 z(\xi)+y(\xi)] h_{e}(\xi-x) d \xi \\
& y(x)=\left[1-\int_{0}^{x} h_{h}(\xi) d \xi\right]+\int_{0}^{x}[2 y(\xi)+z(\xi)] h_{h}(x-\xi) d \xi
\end{aligned}
$$

where $h_{e}(x)$ and $h_{h}(x)$ are the probability density functions (pdf's) of random free-path lengths of the electron and hole, respectively. Let $\alpha$ and $\beta$ be the electron and hole physical ionization coefficients, respectively. Then, a simple hard-dead-space model for the above pdf's is given by ${ }^{7}$

$$
\begin{aligned}
& h_{e}(x)=\alpha e^{-\alpha\left(x-d_{e}\right)} u\left(x-d_{e}\right) \\
& h_{h}(x)=\beta e^{-\beta\left(x-d_{h}\right)} u\left(x-d_{h}\right),
\end{aligned}
$$

where $u(x)$ is the unit step function. The solutions to (1) and (2) are in turn used to determine the mean gain using

$$
\langle G\rangle=\frac{1}{2}(z(0)+1)
$$

The excess noise factor can also be computed using

$$
F=\frac{z_{2}(0)+2 z(0)+1}{(z(0)+1)^{2}}
$$

where $z_{2}(x)$ and $y_{2}(x)$ are the second-order moments which are governed by the following recurrence relations ${ }^{7}$ :

$$
\begin{aligned}
& z_{2}(x)=\left[1-\int_{0}^{w-x} h_{e}(\xi) d \xi\right]+\int_{x}^{w}\left[2 z_{2}(\xi)+y_{2}(\xi)+4 z(\xi) y(\xi)+2 z^{2}(\xi)\right] h_{e}(\xi-x) d \xi \\
& y_{2}(x)=\left[1-\int_{0}^{x} h_{h}(\xi) d \xi\right]+\int_{0}^{x}\left[2 y_{2}(\xi)+z_{2}(\xi)+4 z(\xi) y(\xi)+2 y^{2}(\xi)\right] h_{h}(x-\xi) d \xi
\end{aligned}
$$

The recurrence equations (1), (2), (7) and (8) can be solved, for example, by means of a simple iterative numerical technique.

\subsection{Statistics of the Impulse Response}

Recurrence equations have been previously developed by Hayat et al. for the mean and the variance of the APD impulse response. ${ }^{12}$ These equations involve the electron/hole lifetime pdf's and the carriers' saturation velocities. However, to determine the power spectral density of the APD, which is the Fourier transform of the autocorrelation function of the impulse response, a new set of recurrence equations are needed. One of the main contributions of this paper is to report the development of such recurrence equations and apply them to predict the power spectral density of actual thin devices.

We now review some of the basics of the technique. Following our earlier work, ${ }^{12}$ let $Z_{e}(t, x)$ be the total number of electrons at time $t$ resulting from an initial parent electron at location $x$ and time 0 . 
Similarly, let $Y_{e}(t, x)$ be defined similarly to $Z_{e}(t, x)$ but with the initial electron replaced with a hole. Now define $Z_{h}(t, x)$ as the total number of holes at time $t$ resulting from an initial parent electron at location $x$ and time 0 , and finally, let $Y_{h}(t, x)$ be defined similarly to $Z_{h}(t, x)$ with the initial electron replaced with a hole. To see how these quantities can be used, consider an electron-injected device (electron injected at $x=0$ ) and note that the impulse response $I(t)$ is related to $Z_{e}(t, x)$ and $Z_{h}(t, x)$ through the simple relation

$$
I(t)=q / w\left[v_{e} Z_{e}(t, 0)+v_{h} Z_{h}(t, 0)\right]
$$

The key idea that enables the development of the statistics of $Z_{e}(t, x), Z_{h}(t, x), Y_{e}(t, x)$, and $Y_{h}(t, x)$ is based on a renewal argument. For example, consider the quantity $Z_{e}(t, x)$ and suppose for the moment that an initial parent electron (at $x$ ) first impact ionizes at location $\xi>x$. In this case, there will be two newly created electrons and a hole at the location $\xi$. Each of the two electrons and the hole will independently induce an avalanche process at the new location with a time equal to $t$ less the electron transit time from $x$ to $\xi$. This type of renewal argument is also used to characterize the mean and the variance of $I(t) .{ }^{12}$ In this subsection, we show how this argument is used to derive the autocorrelation function of $I(t)$. We present the key steps and defer the complete derivation and the detailed final recurrence relations to a later time.

Let $R\left(t_{1}, t_{2}\right)$ be the autocorrelation of $I(t)$ defined as $\mathrm{E}\left[I\left(t_{1}\right) I\left(t_{2}\right)\right]$. Clearly, in order to compute the autocorrelation function, it is imperative to calculate the autocorrelations $C_{Z_{e}}\left(t_{1}, t_{2}, x\right)=$ $\mathrm{E}\left[Z_{e}\left(t_{1}, x\right) Z_{e}\left(t_{2}, x\right)\right]$ and $C_{Z_{h}}\left(t_{1}, t_{2}, x\right)=\mathrm{E}\left[Z_{h}\left(t_{1}, x\right) Z_{h}\left(t_{2}, x\right)\right]$ as well as the crosscorrelation $C_{Z}\left(t_{1}, t_{2}, x\right)=$ $\mathrm{E}\left[Z_{e}\left(t_{1}, x\right) Z_{h}\left(t_{2}, x\right)\right]$. For brevity, we will present the recurrence equation only for $C_{Z_{e}}\left(t_{1}, t_{2}, x\right)$.

To do so, we first condition on the location where the parent electron (created at $x$ ) first impact ionizes. Conditional on this location being $\xi$ (note that $\xi \geq x$ ), then the conditional mean of $Z_{e}\left(t_{1}, x\right) Z_{e}\left(t_{2}, x\right)$ given $\xi$, can be written as

$$
\begin{aligned}
\mathrm{E}\left[Z_{e}\left(t_{1}, x\right) Z_{e}\left(t_{2}, x\right) \mid \xi\right] & =\mathrm{E}\left[\left(Z_{e}^{\prime}\left(t_{1}-(\xi-x) / v_{e}, \xi\right)+Z_{e}^{\prime \prime}\left(t_{1}-(\xi-x) / v_{e}, \xi\right)+Y_{e}\left(t_{1}-(\xi-x) / v_{e}, \xi\right)\right)\right. \\
& \left.\times\left(Z_{e}^{\prime}\left(t_{2}-(\xi-x) / v_{e}, \xi\right)+Z_{e}^{\prime \prime}\left(t_{2}-(\xi-x) / v_{e}, \xi\right)+Y_{e}\left(t_{2}-(\xi-x) / v_{e}, \xi\right)\right)\right],
\end{aligned}
$$

where $Z_{e}^{\prime}\left(t_{1}-(\xi-x) / v_{e}, \xi\right)$ and $Z_{e}^{\prime \prime}\left(t_{1}-(\xi-x) / v_{e}, \xi\right)$ correspond to the regenerated and the newlygenerated electrons at $\xi$, respectively, and the term $Y_{e}\left(t_{1}-(\xi-x) / v_{e}, \xi\right)$ corresponds to the hole generated at $\xi$. The delay term $(\xi-x) / v_{e}$ is the parent-electron transit time between positions $x$ and $\xi$. Since the pdf of the electron lifetime is known, we can average (10) over all possible values of $\xi$. Note, however, that the expression in (10) is only valid if both $t_{1}$ and $t_{2}$ exceed the electron transit time to the first impact ionization, given by $t_{1}<(\xi-x) / v_{e}$. For example, if $t_{1} \leq t_{2}$ and $t_{1}<(\xi-x) / v_{e}$, then we must rewrite $(10)$ as

$$
\mathrm{E}\left[Z_{e}\left(t_{1}, x\right) Z_{e}\left(t_{2}, x\right) \mid \xi\right]=\mathrm{E}\left[\left(Z_{e}^{\prime}\left(t_{2}-(\xi-x) / v_{e}, \xi\right)+Z_{e}^{\prime \prime}\left(t_{2}-(\xi-x) / v_{e}, \xi\right)+Y_{e}\left(t_{2}-(\xi-x) / v_{e}, \xi\right)\right)\right]
$$

since in this case, $Z_{e}\left(t_{1}, x\right)=1$. Other similar cases are also possible and must be carefully accounted for. After a careful analysis of all the cases, the following recurrence equation is obtained: for $t_{1} \leq t_{2}$,

$$
\begin{aligned}
C_{Z_{e}}\left(t_{1}, t_{2}, x\right) & =u_{1}\left(t_{2}, x\right)\left[1-H_{e}\left(v_{e} t_{2}\right)\right] \\
& +\int_{\min \left(x+v_{e} t_{1}, w\right)}^{\min \left(x+v_{e} t_{2}\right)}\left\{2 z_{e}\left(t_{2}-\Delta_{1}, s\right)+y_{e}\left(t_{2}-\Delta_{1}, s\right)\right\} h_{e}(s-x) d s
\end{aligned}
$$




$$
\begin{aligned}
& +\int_{x}^{\min \left(x+v_{e} t_{1}, w\right)}\left[2 C_{Z_{e}}\left(t_{1}-\Delta_{1}, t_{2}-\Delta_{1}, s\right)+C_{Y_{e}}\left(t_{1}-\Delta_{1}, t_{2}-\Delta_{1}, s\right)\right. \\
& +\quad 2 z_{e}\left(t_{1}-\Delta_{1}, s\right) z_{e}\left(t_{2}-\Delta_{1}, s\right)+2 z_{e}\left(t_{1}-\Delta_{1}, s\right) y_{e}\left(t_{2}-\Delta_{1}, s\right) \\
& \left.+\quad 2 y_{e}\left(t_{1}-\Delta_{1}, s\right) z_{e}\left(t_{2}-\Delta_{1}, s\right)\right] h_{e}(s-x) d s
\end{aligned}
$$

where $\Delta_{1}=(s-x) / v_{e}$, and $\Delta_{2}=(x-s) / v_{h}$, and the functions $z_{e}(t, x)$ and $z_{e}(t, x)$ are the mean values of $Z_{e}(t, x) Y_{e}(t, x)$, respectively. They can be computed using the recurrence equation for the mean impulse response function. ${ }^{12}$ Finally, the function $H_{e}$ is the cumulative probability distribution corresponding to the pdf $h_{e}$. A similar technique can be used to derive recurrence equation for $C_{Y_{e}}(t, x)$, $C_{Z_{h}}(t, x)$, and $C_{Z}(t, x)$.

\subsection{Breakdown Voltage}

The avalanche breakdown voltage can be defined as the reverse-bias voltage at which the mean gain becomes infinite. To characterize this condition, we undertake an analysis that relies on the asymptotic exponential decay rate of the tail of the mean impulse response function of the APD. ${ }^{12}$ The basic idea of the approach relies on the fact that an exponentially decaying tail of the mean impulse response implies a finite mean gain. Our approach is therefore centered on the observation that the breakdown voltage is precisely the voltage that yields a zero exponential decay rate of the tail of the mean impulse response function. A closed-form expression for the decay rate has been previously developed by the authors and is given by Equations (43) and (38) in. ${ }^{12}$ To find the breakdown voltage, the decay rate must be plotted as a function of the applied reverse bias and the voltage at which the decay rate crosses zero is identified as the breakdown voltage.

\section{Discussion}

The DSMT has been applied to the experimental results obtained from J.C. Campbell for GaAs and AlGaAs and more recently for InP and InAlAs APDs. We were able to fit the ionization coefficients associated with devices of various widths, as a function of the electric field, within the confines of a single exponential model, as shown in Fig. 1 (for brevity, only the electron ionization coefficient for $\operatorname{InP}$ is shown).

The method for calculating the physical ionization coefficients is described as follows: (i) Given the knowledge of the electric field (from the reverse-bias voltage) and the width of the multiplication region along with the ionization threshold energies, the electron and hole dead spaces are computed. (ii) For each measured gain and excess-noise factor pair, appropriate values for $\alpha$ and $\beta$ are determined from the recurrence equations for the gain and the excess noise factor. This step is done by selecting initial values for the ionization coefficients in the recurrence equations and subsequently varying them until the correct (i.e., in agreement with measurement) gain and excess noise factor are obtained. (iii) The previous procedure is repeated for other measured values of the gain and excess noise factor. (iv) When all gain-noise data points are exhausted, the resulting ionization coefficients are fitted in the standard exponential model and the parameters of the exponential model (three for each carrier) are calculated using a least-square-error fit. This completes the first-round calculation of the model for 
carrier ionization coefficients. At this point, one can use the models for the electron and hole ionization coefficients to predict the dependence of the excess-noise factor on the gain. (v) The last step is to repeat all the previous steps (i)-(iv) while slightly adjusting the electron and hole ionization thresholds until the best excess-noise versus gain prediction is obtained. For example, for the case of InP, the initial values for the electron and hole ionization energies were selected according to ${ }^{14}$ and ${ }^{15}$ as $1.84 \mathrm{eV}$ and $1.64 \mathrm{eV}$, respectively, and the optimized values were found to be $2.05 \mathrm{eV}$ and $2.20 \mathrm{eV}$, respectively for electrons and holes. This last step optimizes the model for the ionization coefficients and has significantly improved the accuracy of predicting the noise-gain behavior.

Figure 2 shows the DSMT predictions and the experimental results for InP APDs of varying thickness. It is clear that the DSMT correctly predicts of the reduction in the excess noise factor in thin devices. Moreover, in conjunction with the width-independent ionization coefficients presently available, the DSMT characterization of the statistics of the APD impulse response can be used to predict the statistics of the frequency response. Our results show that as the multiplication-region thickness decreases, the DSMT estimate of the 3-dB bandwidth becomes significantly lower than that obtained from the conventional multiplication theory estimate (which uses the ionization coefficients for bulk material), as shown in Figs. 3 and 4. For example, as seen in Fig. 4, a reduction of about 30\% in the $3-\mathrm{dB}$ bandwidth is predicted for a 100-nm GaAs device. As expected, the discrepancy between the DSMT and the conventional theory becomes negligible when the thickness of the multiplication region large (e.g., in excess of $500 \mathrm{~nm}$ ).

Fluctuations in the frequency response can also be estimated. This is done by computing the power spectral density of the impulse response. Figure 5 shows the DSMT prediction of the power-spectral density for a thin APD overlayed with the mean frequency response. Further analysis indicates that the relative spectral fluctuations become smaller as the thickness is reduced. This added property of thin APDs alludes to improved APD performance in high-speed detection applications. It must be noted, however, that the conventional theory does not predict such an improved spectral performance. This is akin to the inability of the conventional theory to predict the reduced excess noise factor in thin devices.

Finally, to predict the breakdown voltage, the exponential model for the ionization coefficients for each material is used in the DSMT to determine the decay rate of the tail of the mean impulse response. The decay rate must be plotted as a function of the applied reverse bias. Our preliminary calculations indicate that for thin devices, the DSMT-prediction of the breakdown voltage is higher than that predicted by the conventional theory.

\section{Conclusion}

We reported an improved technique for calculating models for the electron and hole ionization coefficients in GaAs, AlGaAs, InP, and InAlAs APDs. For each material, these models were shown to be independent of the width of the multiplication region of each device. The improvement in the model is achieved by means of optimizing the model parameters over the theoretical ionization threshold energies. Using these models in conjunction with the dead-space multiplication theory, we correctly predicted the gain-noise characteristics of the above APDs for a variety of multiplication-region widths. The frequency response and the power-spectral density were also computed in the confines of the deadspace multiplication theory. It is clear that for thin APDs, the effect of dead space is significant on 
both the gain-noise characteristics as well as the fluctuations of the frequency response, especially at high frequencies. This latter effect may have an impact on reducing intersymbol interference (and hence improving the bit-error rate) in high-speed applications.

\section{Aknowledgement}

The authors are thankful to Professor Joe C. Campbell for providing all the experimental data used in this paper. We are also thankful to Dr. Paul Sotirelis for assisting us with high-performance computing issues and facilitating the use of the ASC/MSRC computing facilities located the Wright-Patterson Air Force Base, Ohio.

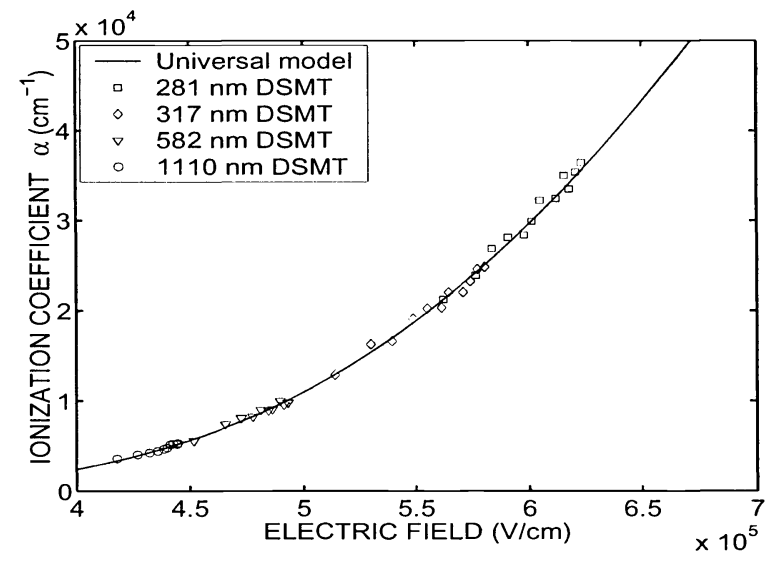

Figure 1: Electron ionization coefficient $(\alpha)$ of InP as a function of the electric field.

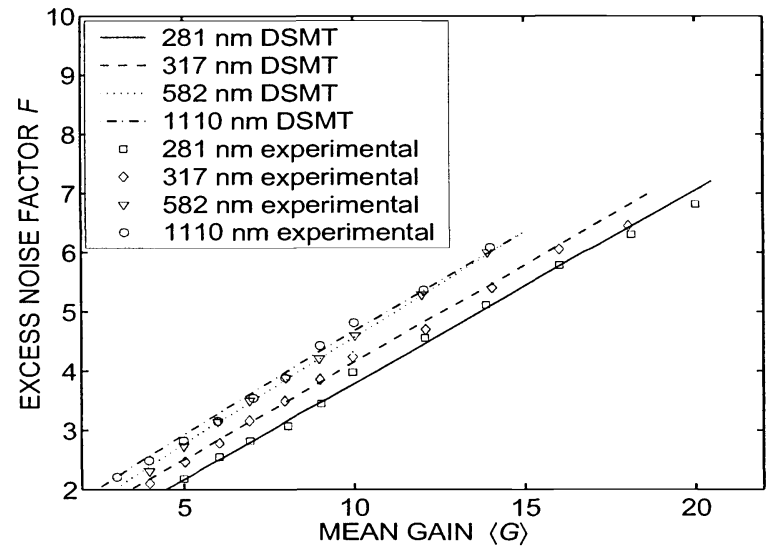

Figure 2: The DSMT predictions of the gain vs. noise characteristics for thin InP APDs. 


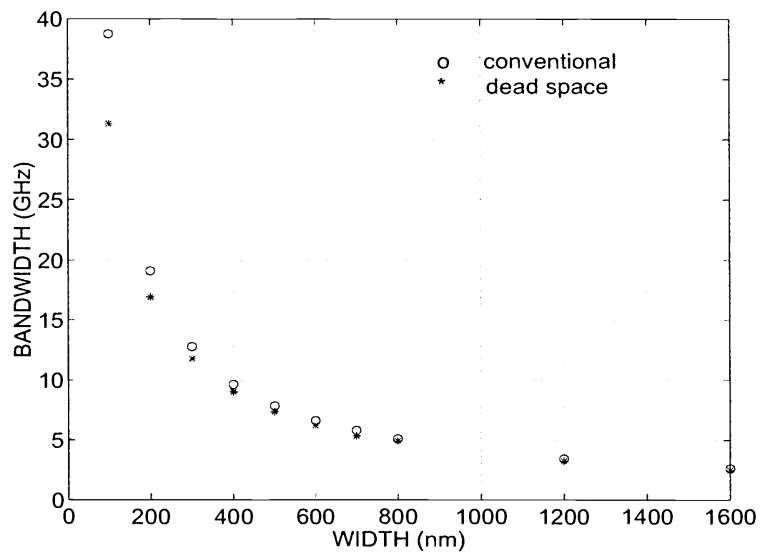

Figure 3: Comparison between the conventional and DSMT predictions of the $3-\mathrm{dB}$ bandwidth for GaAs APDs of varying thickness.

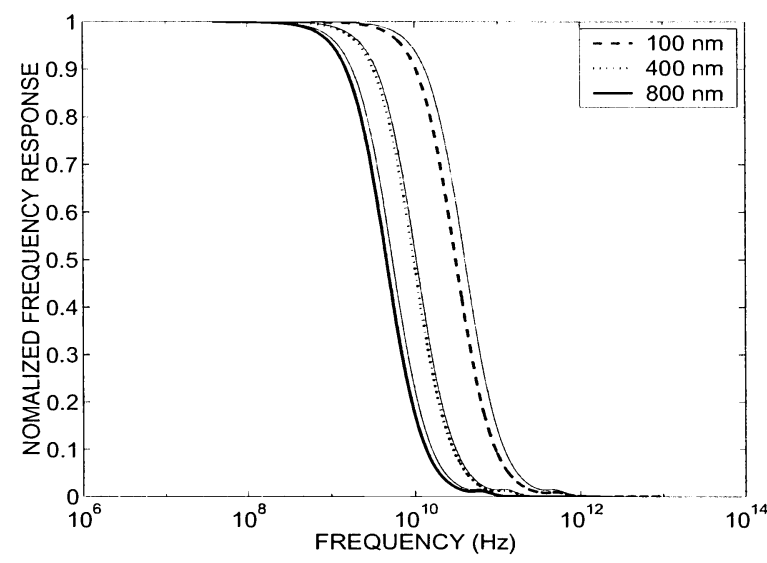

Figure 4: Conventional and DSMT predictions of the frequency response for three devices: $100 \mathrm{~nm}, 400 \mathrm{~nm}$, and $800 \mathrm{~nm}$ GaAs APDs.

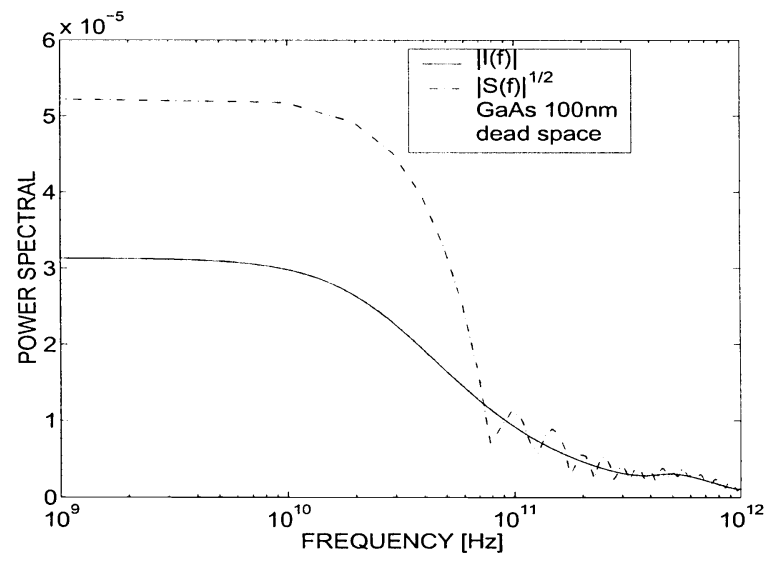

Figure 5: Power-spectral density of a $100 \mathrm{~nm}$ GaAs APD along with the frequency response. 


\section{REFERENCES}

[1] R. J. McIntyre, "Multiplication noise in uniform avalanche photodiodes." IEEE Trans. Elect. Dev., vol. ED13, pp. 164 168, 1966.

[2] J. C. Campbell, S. Chandrasekhar, W. T. Tsang, G. J. Qua, and B. C. Johnson, "Multiplication noise of wide-bandwidth InP/InGaAsP/InGaAs avalanche photodiodes," J. Lightwave Technol., vol. 7, pp. 473 477, 1989.

[3] V. Chandramouli, C. M. Maziar, and J. C. Campbell, "Design considerations for high performance avalanche photodiode multiplication layers," IEEE Trans. Electron Dev., vol. 41, pp. 648-654, 1994.

[4] C. Hu, K. A. Anselm, B. G. Streetman, and J. C. Campbell, "Noise characteristics of thin multiplication region GaAs avalanche photodiodes," Appl. Phys. Lett., vol. 69, pp. 3734-3736, 1996.

[5] C. Lenox, P. Yuan, H. Nie, O. Baklenov, C. Hansing, J. C. Campbell, A. L. Holmes, Jr., and B. G. Streetman, "Thin multiplication region InAlAs homojunction avalanche photodiodes," Appl. Phys. Lett., vol. 73 , pp. $783-784,1998$.

[6] K. A. Anselm, P. Yuan, C. Hu, C. Lenox, H. Nie, G. Kinsey, J. C. Campbell, and B. G. Streetman, "Characteristics of GaAs and AlGaAs homojunction avalanche photodiodes with thin multiplication regions," Appl. Phys. Lett., vol. 71, pp. 3883-3885, 1997.

[7] M. M. Hayat, B. E. A. Saleh, and M. C. Teich, "Effect of dead space on gain and noise of double-carriermultiplication avalanche photodiodes," IEEE Trans. Elect. Dev., vol. 39, pp. 546-552, 1992.

[8] M. M. Hayat, W. L. Sargeant, and B. E. A. Saleh, "Effect of dead space on gain and noise in Si and GaAs avalanche photodiodes," IEEE J. Quantum Electron., vol. 28, pp. 1360-1365, 1992

[9] M. A. Saleh, M. M. Hayat, B. E. A. Saleh, and M. C. Teich, "Dead-space-based theory correctly predicts excess noise factor for thin GaAs and AlGaAs APDs," IEEE Trans. Elect. Dev., vol. 47, pp. 625-633, 2000.

[10] P. Yuan, K. A. Anselm, C. Hu, H. Nie, C. Lenox, H. L. Holmes, B. G. Streetman, J. C. Campbell, and R. J. McIntyre, "A new look at impact ionization-Part II: Gain and noise in short avalanche photodiodes," IEEE Trans. Electron. Dev., vol. 46, pp. 1632-1639, 1999.

[11] P. Yuan, C. C. Hansing, K. A. Anselm, C. V. Lenox, H. Nie, H. L. Holmes, B. G. Streetman, and J. C. Campbell, "Impact ionization characteristics of III-V semiconductors for a wide range of multiplication region thicknesses," IEEE J. Quantum Electron., vo. 36, no. 2, pp. 198-204, 2000.

[12] M. M. Hayat and B. E. A. Saleh, "Statistical properties of the impulse response function of double-carrier multiplication avalanche photodiodes including the effect of dead space," J. Lightwave Technol., vol. 10, pp. 1415-1425, 1992.

[13] Y. Okuto and C. R. Crowell, "Ionization coefficients in semiconductors: A nonlocalized property," Phys. Rev. B, vol. 10, pp. 4284-4296, 1974.

[14] T. P. Pearsall, "Threshold energies for impact ionization by electrons and holes in InP," Appl. Phys. Lett., vol. 35, pp. 168-170, 1979.

[15] I. Watanabe, T. Toshitaka, K. Taguchi, "Monte carlo simulation of impact ionization rates in InAlAs-InGaAs Square and graded barrier superlattice ,"IEEE J. Quantum Electron., vol. 31, no. 10 pp. 1826-1834, 1995. 